\title{
Is the prevalence of coronary heart disease falling in British men?
}

\author{
F C Lampe, R W Morris, P H Whincup, M Walker, S Ebrahim, A G Shaper
}

\begin{abstract}
Objective-To assess whether long term trends over time in acute coronary heart disease (CHD) event rates have influenced the burden of prevalent CHD in British men.

Design-Longitudinal cohort study.

Participants-7735 men, aged 40-59 at entry (1978-80), selected from 24 British towns.

Methods-The prevalences of current angina symptoms and history of diagnosed CHD were ascertained by questionnaire in 1978-80, 1983-85, 1992, and 1996. New major CHD events (fatal and non-fatal) were ascertained throughout the study from National Health Service central registers and general practice record reviews. Age adjusted trends in CHD prevalence were compared with trends in major CHD event rates.

Results-From 1978-1996 there was a clear decline in the prevalence of current angina symptoms: the age adjusted annual percentage change in odds was $-1.8 \%$ (95\% confidence interval $(\mathrm{CI})-2.8 \%$ to $-0.8 \%$ ). However, there was no evidence of a trend in the prevalence of history of diagnosed CHD (annual change in odds $0.1 \%, 95 \% \mathrm{CI}-1.0 \%$ to $1.2 \%$ ). Over the same period, the CHD mortality rate fell substantially (annual change $-4.1 \%, 95 \%$ CI $-6.5 \%$ to $-1.6 \%$ ); rates of non-fatal myocardial infarction, all major CHD events, and first major CHD event fell by $-1.7 \%$ (95\% CI $-3.9 \%$ to $0.5 \%),-2.5 \%$ ( $95 \%$ CI $-4.1 \%$ to $-0.8 \%)$, and $-2.4 \%(95 \% \mathrm{CI} \%-4.3$ to $-0.4 \%)$, respectively.

Conclusions-These results suggest that middle aged British men are less likely to experience symptoms of angina than in previous decades but are just as likely to have a history of diagnosed CHD. Despite falling rates of new major events and falling symptom prevalence, the need for secondary prevention among middle aged men with established CHD is as great as ever.

(Heart 2001;86:499-505)
\end{abstract}

Keywords: coronary heart disease; angina; prevalence; trends

Deaths from coronary heart disease (CHD) have fallen in recent decades in most of the industrialised world, including the UK. ${ }^{1} \mathrm{Re}-$ cent results from the MONICA (monitoring trends and determinants in cardiovascular disease) project show that in most populations, a fall in the rate of new major CHD events is the primary contributor to declining CHD mortality, although a fall in case fatality has also had a substantial impact. ${ }^{2}$ However, the extent to which, or even the direction in which, these changes have affected the burden of CHD in the population has not been established. It has been suggested that improved survival after myocardial infarction (MI) may actually outweigh falling incidence of new events, leading to an increase in disease prevalence and therefore a greater population burden of serious morbidity and increased treatment need..$^{3-5}$ Using data from a longitudinal cohort study, we have examined time trends in the prevalence of current angina symptoms and history of diagnosed CHD from 1978 to 1996 in a representative sample of British middle aged men and compared these with trends in the rate of occurrence of new major CHD events in the same population.

\section{Methods}

The British Regional Heart Study is a prospective study of 7735 men aged $40-59$ years at entry (1978-80), who were randomly selected from the age and sex registers of one general practice in each of 24 British towns. Men with pre-existing CHD were not excluded. Selection procedures have been described in detail. ${ }^{6}$ In brief, the 24 towns were taken from those with populations of 50 000-100 000 (1971 census); they covered the full range of mortality from cardiovascular disease and included all major geographical regions. The general practice in each town was required to have a social class distribution representative of that town. The baseline assessment included a nurse administered questionnaire $(\mathrm{Q} 1)$. The overall response rate was $78 \%$. Three additional postal questionnaires were sent to surviving men: firstly, at the fifth anniversary of study entry 1983-85 (Q5); secondly, in November 1992 (Q92); and thirdly, in November 1996 (Q96). In two of the 24 towns, Q96 was sent in February and April 1996 rather than in November, as part of a pilot study for a subsequent re-examination of the men. Response rates for Q5, Q92, and Q96 were $98 \%, 91 \%$, and $88 \%$, respectively. The men were also followed up from baseline through National Health Service central registers for mortality and through biennial reviews of their general practice medical records (including hospital correspondence) for nonfatal cardiovascular events. ${ }^{7}$ By 31 December $1995,99 \%$ of the 7735 men initially examined in 1978-80 had been followed up for 15 years; the remaining $1 \%$ were lost to follow up.

\section{DEFINITIONS OF CHD PREVALENCE}

Two questionnaire measures of prevalent CHD were chosen. Each was defined at Q1, Q5, Q92, 
Table 1 Prevalence of current angina symptoms and history of diagnosed coronary heart disease (CHD) by age and questionnaire

\begin{tabular}{|c|c|c|c|c|c|c|c|c|c|}
\hline & \multicolumn{8}{|c|}{ Age group (years) } & \multirow[b]{2}{*}{ All men } \\
\hline & $40-45$ & $45-49$ & $50-54$ & $55-59$ & $60-64$ & $65-69$ & $70-74$ & $75-79$ & \\
\hline \multicolumn{10}{|c|}{ Total number of men } \\
\hline Q1 (1978-80) & 1838 & 1898 & 1974 & 2025 & & & & & 7735 \\
\hline Q5 (1983-85) & & 1761 & 1821 & 1861 & 1832 & & & & 7275 \\
\hline Q92 (1992) & & & 421 & 1587 & 1533 & 1445 & 939 & & 5925 \\
\hline Q96 (1996) & & & & 701 & 1482 & 1373 & 1179 & 528 & 5263 \\
\hline \multicolumn{10}{|l|}{ Mean age (years) } \\
\hline Q1 (1978-80) & 42.5 & 47.5 & 52.5 & 57.6 & & & & & 50.2 \\
\hline Q5 (1983-85) & & 47.5 & 52.5 & 57.5 & 62.6 & & & & 55.1 \\
\hline Q92 (1992) & & & 54.2 & 57.5 & 62.5 & 67.4 & 71.8 & & 63.3 \\
\hline Q96 (1996) & & & & 58.7 & 62.5 & 67.5 & 72.4 & 76.3 & 66.9 \\
\hline \multicolumn{10}{|c|}{ Prevalence of current angina symptoms (\%) } \\
\hline Q1 (1978-80) & 4.0 & 6.1 & 9.2 & 11.7 & & & & & 7.8 \\
\hline Q5 (1983-85) & & 6.6 & 7.5 & 13.2 & 15.7 & & & & 10.8 \\
\hline Q92 (1992) & & & $8.1^{\star}$ & 9.6 & 13.7 & 16.4 & $18.5^{\star}$ & & 13.6 \\
\hline Q96 (1996) & & & & $9.7^{\star}$ & 11.5 & 14.5 & 16.2 & 18.3 & 13.8 \\
\hline \multicolumn{10}{|c|}{ Prevalence of history of diagnosed CHD (angina or MI) (\%) } \\
\hline Q1 (1978-80) & 1.6 & 3.2 & 6.8 & 9.8 & & & & & 5.5 \\
\hline Q5 (1983-85) & & 3.2 & 6.3 & 10.7 & 13.5 & & & & 8.5 \\
\hline Q92 (1992) & & & $8.3^{\star}$ & 9.7 & 15.6 & 21.1 & $23.8^{\star}$ & & 16.1 \\
\hline Q96 (1996) & & & & $10.2^{\star}$ & 13.3 & 19.2 & 20.9 & 26.8 & 17.5 \\
\hline
\end{tabular}

${ }^{\star}$ Mean age is not equivalent to mean age in cells directly above and below.

MI, myocardial infarction; Q1, nurse administered questionnaire at baseline assessment; Q5, postal questionnaire at fifth anniversary of study entry; Q92, postal questionnaire in November 1992; Q96, postal questionnaire in November 1996.

and Q96. Current angina symptoms were defined as a positive response to either of two standard World Health Organization (Rose) questionnaire items on current chest pain brought on by walking uphill or hurrying, or by walking at an ordinary pace on the level. ${ }^{8}$ Alternative definitions requiring additional WHO (Rose) criteria yielded consistently lower prevalences but had very little or no effect on the magnitude of time trends. History of diagnosed CHD was defined as subject recall of ever having had a doctor's diagnosis of either angina or heart attack (MI, coronary thrombosis). ${ }^{9}$ The first measure was intended to identify men who were currently experiencing disabling symptoms of CHD (whether diagnosed or not) while the second was a measure of lifelong history, identifying men who had ever had an established diagnosis of CHD (whether currently symptomatic or not). The wording of questions and coding schemes were the same for each questionnaire. Angina symptom status was missing for 1, 165, 47, and 212 men at Q1, Q5, Q92, and Q96 respectively, and diagnosed CHD status for 9, 0, 6, and 23 men.

DEFINITIONS OF MAJOR CHD EVENTS

The occurrence of new major CHD events (first or recurrent) was ascertained during 15 years' equal follow up for each subject. Four measures were considered. (1) CHD death was defined as International Classification of Diseases, ninth revision (ICD-9), codes 410-414 according to the underlying cause given on the death certificate, unless this was contraindicated by medical history or necropsy finding. Sudden death for which no other cause was apparent was included if certified to be the result of CHD. (2) A non-fatal $M I$ was defined as an event associated with at least two of the following features at the time of event: history of severe prolonged chest pain; electrocardiographic evidence of $\mathrm{MI}$; and cardiac enzyme abnormalities, with survival for at least 28 days after the onset of symptoms. ${ }^{7}$ (3) A major CHD event was defined as a CHD death or a non-fatal MI. (4) First major CHD event applied only to subjects who did not report doctor-diagnosed heart attack at baseline and was defined as the first major CHD event that occurred during follow up.

STATISTICAL METHODS

Prevalence trends were examined using logistic regression. Age specific models were fitted for the three, five year age bands (50-54, 55-59, and 60-64 years) that were represented in at least three of the four questionnaires. Each subject appeared only once in each age specific analysis, with the exception of a small proportion who were included in both Q92 and Q96 data because of the four year interval between these questionnaires (results were essentially unchanged by exclusion of these subjects from either Q92 or Q96). An overall estimate of trend was obtained by fitting a logistic generalised estimating equation for binary data to the entire data set using PROC GENMOD in SAS (version 6.12; SAS Institute, Cary, North Carolina, USA). This model allowed for association between individual subjects' repeated responses by using an autoregressive correlation structure. Both the age specific models and the overall generalised estimating equation model were adjusted for age; the overall model was additionally adjusted for age squared. The annual linear trend in the log odds of CHD was estimated by including the date of questionnaire as a continuous variable. Prevalence trends were expressed as the age adjusted annual percentage change in odds with $95 \%$ confidence interval (CI). For the prevalences considered in this paper, the change in odds is virtually equivalent to the change in prevalence itself. The estimates of trend are relative (not absolute) percentage changes. To assess trends in major CHD event rates, the follow up time for each subject was divided into three exact five year periods starting from (a) the baseline date, 1978-80; (b) 1983-85; and (c) 1988-90. The occurrence in each time period of the four separate outcomes was considered: (1) CHD 

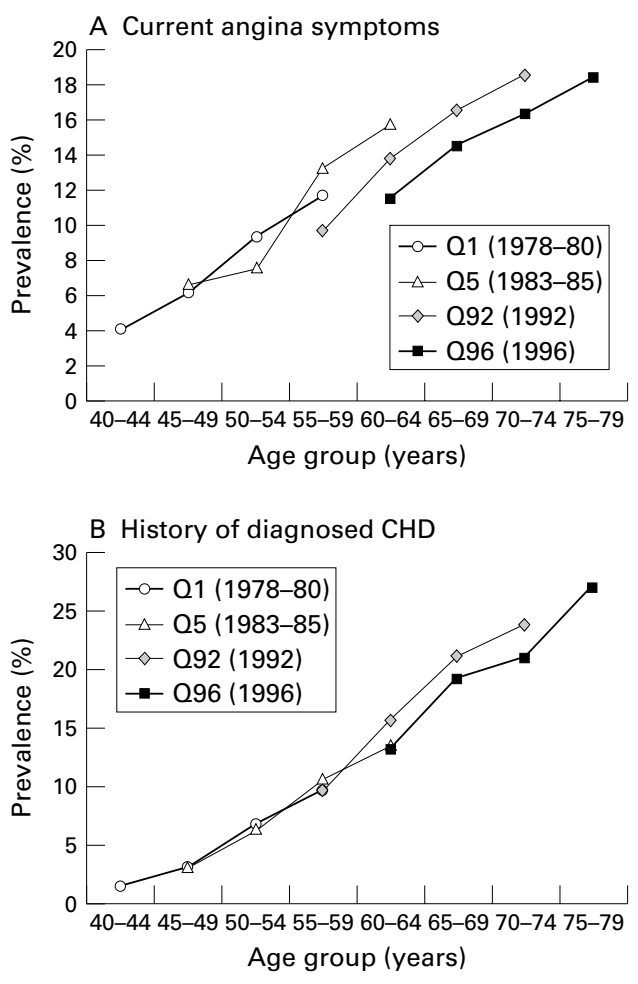

Figure 1 Prevalence of coronary heart disease (CHD) by age and questionnaire. (A) Current angina symptoms. (B) History of diagnosed CHD.

death; (2) number of non-fatal MIs; (3) number of major CHD events; (4) first major $\mathrm{CHD}$ event. Poisson regression was used to examine trends in event rates using date of follow up period, adjusted for age and age squared, and including person time of follow up as an offset. For outcomes 2 and 3 a Poisson generalised estimating equation was used because each subject could have experienced more than one event, and continued to be at risk of an event until death. Trends over time were expressed as age adjusted annual (relative) percentage change in rates with $95 \% \mathrm{CI}$. The annual trend in case fatality (the proportion of major events that were fatal) was assessed for the first major CHD event only, using logistic regression with adjustment for age at the time of event. Social class at baseline was defined on the basis of longest held occupation and grouped as non-manual (I, II, III non-manual) or manual (III manual, IV, V). Tests of interaction were used to assess whether trends in prevalence and event rates differed between the two social class groups. Adjustment for town of recruitment at baseline made little difference to trend estimates; unadjusted results are presented here.

\section{Results}

Table 1 shows the age distribution of the cohort at each questionnaire. The numbers of subjects appearing in both Q92 and Q96 in the same age group were 299, 295, 262, and 217 for age groups 55-59, 60-64, 65-69, and 70-74, respectively. Table 1 also shows the age specific prevalences of current angina symptoms and history of diagnosed CHD. The rows of each table show the effect of age on prevalence at each time point; the columns show the effect of time on prevalence for each age group. The relations are shown graphically in fig 1 . In the absence of secular trends, the four lines representing the four questionnaires would be expected to form a single line showing the association between age and prevalence.

\section{TRENDS IN THE PREVALENCE OF CURRENT} ANGINA SYMPTOMS

It can be seen that the age specific prevalence of current angina symptoms tended to decrease over time. This decrease was apparent from Q5 to Q92, and from Q92 to Q96 (table 1, fig 1). Table 2 shows the age specific and overall estimates of trend. Angina symptom prevalence fell significantly in each of the three age groups. The overall age adjusted annual change (estimated using the entire data set and taking into account the repeated observations on individual subjects) was $-1.8 \%$ ( $95 \%$ CI $-2.8 \%$ to $-0.8 \%$; $\mathrm{p}<0.001)$. In other words, from 1978 to 1996 the odds of a middle aged man having angina symptoms decreased on average by $1.8 \%$ per year. This average fall in prevalence was caused by a decline from Q5 onwards (table 2). After taking this into account, there was no evidence that the trend in symptom prevalence differed by age $(p=0.83$ for interaction between age and time trend).

TRENDS IN THE PREVALENCE OF HISTORY OF DIAGNOSED CHD

The prevalence of diagnosed CHD increased notably with age from less than $2 \%$ among men aged $45-49$ to over $25 \%$ among men aged 75-79 (table 1 and fig 1), but no clear trend over time in prevalence was apparent. Logistic regression analyses showed small nonsignificant annual changes in the prevalence of

Table 2 Time trends in the prevalence of current angina symptoms and history of diagnosed CHD

\begin{tabular}{|c|c|c|c|c|c|c|}
\hline \multirow[b]{2}{*}{ Age group (years) } & \multicolumn{3}{|c|}{ Current angina symptoms } & \multicolumn{3}{|c|}{ History of diagnosed CHD } \\
\hline & $N$ & $n$ & Annual \% change in odds $(95 \% \text { CI })^{\star}$ & $N$ & $n$ & Annual $\%$ change in odds $(95 \% \text { CI })^{*}$ \\
\hline $50-54 \dagger$ & 4182 & 350 & $-2.9(-5.5$ to -0.2$)$ & 4213 & 284 & $-0.4(-3.3$ to 2.5$)$ \\
\hline $55-59 \ddagger$ & 6089 & 694 & $-1.9(-3.1$ to -0.6$)$ & 6169 & 623 & $-0.3(-1.6$ to 1.0$)$ \\
\hline $60-64 \rrbracket$ & 4732 & 653 & $-2.5(-4.0$ to -1.0$)$ & 4839 & 683 & $0.4(-1.1$ to 1.9$)$ \\
\hline All men (40-78) & 25773 & 2871 & $-1.8(-2.8$ to -0.8$)$ & 26160 & 2913 & $0.1(-1.0$ to 1.2$)$ \\
\hline \multicolumn{7}{|l|}{ Specific time periods } \\
\hline Q1 to Q5 (1978-1985) & & & 0.5 (-1.6 to 2.6$)$ & & & $0.6(-1.5$ to 2.8$)$ \\
\hline Q5 to Q92 (1985-1992) & & & $-2.0(-3.4$ to -0.7$)$ & & & $1.9(0.5$ to 3.3$)$ \\
\hline Q92 to Q96 (1992-1996) & & & $-2.4(-4.5$ to -0.3$)$ & & & $-1.4(-3.0$ to 0.2$)$ \\
\hline
\end{tabular}

$\mathrm{N}$, number of observations; $\mathrm{n}$, number positive for condition.

*Age specific estimates determined by logistic regression adjusted for age and overall estimate by logistic generalised estimating equation adjusted for age and age squared, allowing for within subject correlation. Includes: †Q1, Q5, and Q92; †Q1, Q5, Q92, and Q96; \Q5, Q92, and Q96. 
Table 3 Major CHD event rates (/1000/year) by age group and time period

\begin{tabular}{|c|c|c|c|c|c|c|c|}
\hline \multirow[b]{2}{*}{ Year at start of five year period } & \multicolumn{6}{|c|}{ Age group at the start of five year period (years) } & \multirow[b]{2}{*}{ All men (total events) } \\
\hline & $40-45$ & $45-49$ & $50-54$ & $55-59$ & $60-64$ & $65-69$ & \\
\hline \multicolumn{8}{|l|}{ Total number of men } \\
\hline $1978-80$ & 1838 & 1898 & 1974 & 2025 & & & 7735 \\
\hline $1983-85$ & & 1820 & 1855 & 1901 & 1878 & & 7454 \\
\hline $1988-90$ & & & 1788 & 1788 & 1777 & 1637 & 6990 \\
\hline \multicolumn{8}{|l|}{ CHD mortality rate } \\
\hline $1978-80$ & 0.4 & 1.9 & 3.4 & 6.7 & & & $3.2(121)$ \\
\hline $1983-85$ & & 1.4 & 3.5 & 5.7 & 10.7 & & $5.3(192)$ \\
\hline $1988-90$ & & & 2.3 & 5.0 & 7.4 & 9.5 & $5.9(198)$ \\
\hline \multicolumn{8}{|l|}{ Non-fatal MI event rate } \\
\hline $1978-80$ & 2.6 & 5.3 & 6.8 & 7.0 & & & $5.5(209)$ \\
\hline $1983-85$ & & 2.8 & 6.4 & 8.1 & 7.6 & & $6.2(225)$ \\
\hline $1988-90$ & & & 5.0 & 6.3 & 7.2 & 6.9 & $6.3(212)$ \\
\hline \multicolumn{8}{|l|}{ Total major CHD event rate } \\
\hline $1978-80$ & 3.1 & 7.2 & 10.2 & 13.7 & & & $8.7(330)$ \\
\hline $1983-85$ & & 4.2 & 9.9 & 13.9 & 18.3 & & $11.5(417)$ \\
\hline $1988-90$ & & & 7.3 & 11.3 & 14.7 & 16.4 & $12.2(410)$ \\
\hline \multicolumn{8}{|l|}{ First major CHD event rate } \\
\hline $1978-80$ & 2.6 & 5.7 & 7.6 & 10.9 & & & $6.7(243)$ \\
\hline 1983-85 & & 3.6 & 7.6 & 11.0 & 14.1 & & $9.0(304)$ \\
\hline $1988-90$ & & & 6.3 & 8.1 & 12.0 & 13.4 & $9.7(301)$ \\
\hline
\end{tabular}

^Excludes 300 (of 7735) men with a history of diagnosed MI (or missing information) at baseline.

diagnosed CHD in each of the three age groups (table 2). Overall, the age adjusted annual change in odds estimated from the entire data set was $0.1 \%(95 \%$ CI $-1.0 \%$ to $1.2 \%$; $\mathrm{p}=0.83)$.

SOCIAL CLASS AND PREVALENCE TRENDS

Men belonging to the manual social class at study entry had almost double the prevalence of angina symptoms as those from the non-manual group, although this social class difference narrowed as age increased. The time trend in angina symptom prevalence was seen in both social class subgroups. The decrease appeared slightly stronger for the non-manual subgroup, for whom the annual change in odds was $-2.1 \% \quad(95 \%$ CI $-3.9 \%$ to $-0.3 \%)$ compared with $-1.3 \%(95 \%$ CI $-2.6 \%$ to $-0.03 \%$ ) for the manual subgroup, but this social class difference in trend was not significant. ( $\mathrm{p}=0.45$ for interaction).

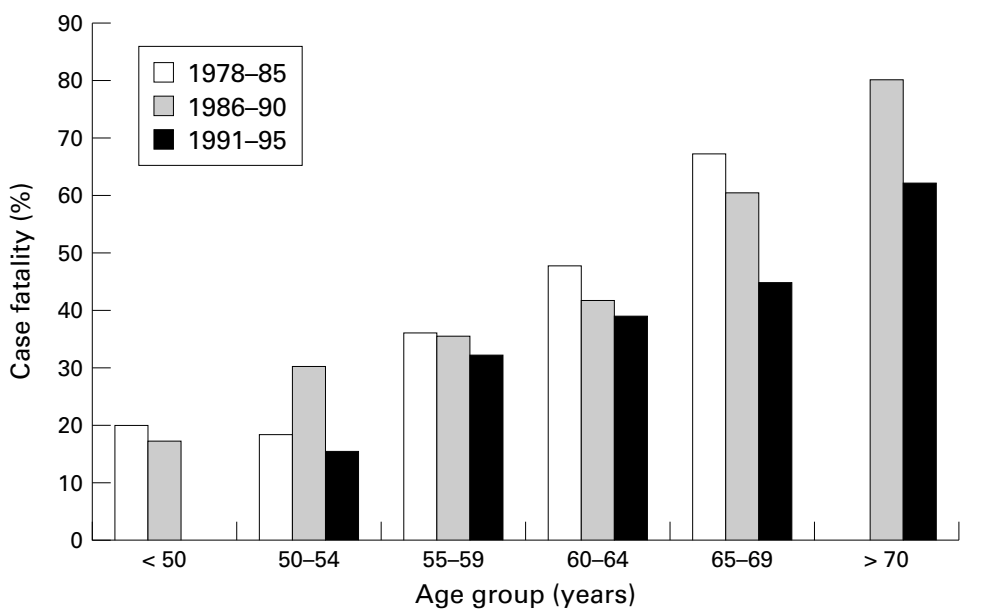

$\begin{array}{lcccccc}\begin{array}{l}\text { Fatal first } \\ \text { events/ } \\ \text { total first }\end{array} & 11 / 57 & 36 / 163 & 67 / 193 & 111 / 261 & 94 / 178 & 63 / 99 \\ \text { events } & & & & & & \\ \begin{array}{l}\text { Case } \\ \text { fatality (\%) }\end{array} & 19.3 & 22.1 & 34.7 & 42.5 & 52.8 & 63.6\end{array}$

Figure 2 Case fatality (proportion of events fatal within 28 days) for first major CHD event by age at event and year of event.
TRENDS IN RATES OF CHD MORTALITY AND MAJOR CHD EVENTS

During 15 years of equal follow up from baseline, 985 of the 7735 men experienced a new major CHD event and 511 men died from CHD. Counting recurrent events for a single subject, there were 646 non-fatal MIs and 1157 major CHD events in total. Of 7435 men without a history of diagnosed MI at baseline, 848 had a first major CHD event. Table 3 shows the age specific rates of CHD mortality, non-fatal MIs, all major CHD events, and first major CHD event for the three, five year follow up periods. Event rates tended to fall over time in all age groups and for each of the four outcomes. Overall, the age adjusted annual changes were $-4.1 \%(95 \% \mathrm{CI}-6.5 \%$ to $-1.6 \%)$ for CHD mortality $(p=0.002),-1.7 \%(95 \%$ CI $-3.9 \%$ to $0.5 \%)$ for non-fatal MIs $(\mathrm{p}=0.12),-2.5 \%(95 \% \mathrm{CI}-4.1 \%$ to $-0.8 \%)$ for total events $(\mathrm{p}=0.004)$, and $-2.4 \%(95 \%$ CI $-4.3 \%$ to $-0.4 \%)$ for first event $(p=0.015)$. The relative reduction in all major CHD events was very similar in non-manual and manual social class groups $(-2.3 \% v-2.6 \%)$.

\section{TRENDS IN CASE FATALITY}

Case fatality was examined for first major CHD events only, using all available follow up (baseline to 31 December 1995). Of 7435 men without a history of MI at baseline, 951 had a first major CHD event during this follow up period, of which 382 were fatal within 28 days. Figure 2 shows the case fatality proportion by age at event and year of event. Case fatality rose substantially with increasing age but tended to fall over time within each age group. The overall annual age adjusted change in the odds of a fatal event was $-3.2 \%$ (95\% CI $-6.8 \%$ to $0.5 \%$; $\mathrm{p}=0.089)$. The fall was more pronounced in men of non-manual than of manual social class $(-7.3 \% v-2.2 \%$, respectively), but a test for interaction was not significant $(p=0.35)$. It should be noted that, as case fatality rates are high, the odds ratio is not a good estimate of the relative risk. The age adjusted relative change in the case fatality proportion itself was 
estimated from Poisson regression analysis to be $-2.1 \%(95 \%$ CI $-5.0 \%$ to $0.8 \%)$.

\section{Discussion}

There is now little doubt that the falls in CHD mortality seen in many western countries since the 1960 s or 1970 s are real. ${ }^{2}$ Numerous reports have documented changes in CHD mortality and major events ${ }^{10-15}$ but the effect on CHD prevalence has not been established. We have examined trends in new major events together with trends in prevalence in a representative cohort of British men. CHD mortality, major CHD events, and case fatality fell from 1978 to 1996. Angina symptom prevalence fell in parallel with acute event rates but the prevalence of history of diagnosed CHD remained stable.

\section{METHODOLOGICAL ISSUES}

A potential source of bias in our study was the change from use of an administered questionnaire initially to postal questionnaires for subsequent assessment. Some studies have suggested that the self administered WHO chest pain questionnaire yields higher estimates of Rose angina prevalence. ${ }^{16}{ }^{17}$ If this is so, then our study may have underestimated the true decline in symptom prevalence from Q1 to Q96 as a clear fall occurred from Q5 to Q96, where identical (self administered) questionnaires were used. The trend in angina symptom prevalence was independent of the precise definition used; with the standard WHO (Rose) definition the annual change was $-2.0 \%$. The use of a chest pain questionnaire to measure trends in angina symptom prevalence should ensure independence from changes in diagnostic patterns and is likely to be more appropriate than the use of other methods such as clinical opinion, which are difficult to standardise over time. We used self reported history as a measure of lifelong diagnosis of CHD as information from medical record reviews was available for only the study period. In this study population there is very high agreement between self report and medical record for diagnosed $\mathrm{CHD}^{18}$ and the between questionnaire consistency rates for a positive report of diagnosed CHD range from $82-94 \%$. Differing rates of non-response to each of the four questionnaires may introduce bias. Compared with responders, non-responders to Q1 had a slightly increased five year death rate. ${ }^{19}$. Non-responders to the three postal questionnaires had a slightly (1.1-1.4-fold) increased prevalence of CHD symptoms and diagnoses at previous questionnaires. Simple sensitivity analyses suggested that these differences were unlikely to have a significant impact on the estimated prevalence trends.

TRENDS IN MAJOR CHD EVENTS

Among these middle aged British men, the substantial fall in CHD mortality was caused both by a fall in the rate of new major events and by declining case fatality. The magnitudes of the trends found in this study are quite similar to the overall results for men reported from MONICA (1980s to 1990s): relative annual changes were $-4.0 \%$ for official CHD mortality, $-2.7 \%$ for MONICA CHD mortality, $-2.1 \%$ for
MONICA major events, $-2.1 \%$ for non-fatal $\mathrm{MI}$, and $-0.6 \%$ for case fatality. ${ }^{2}$ Although MONICA CHD mortality fell less than that based on death certificates only, the two rate reductions were very similar in the UK centres (Glasgow and Belfast). There are no previous reports of trends in major CHD events from representative English populations. It is likely that both changes in risk factors and improvements in medical care have influenced trends in event rates and case fatality, ${ }^{20}{ }^{21}$ but the relative effect of these two factors on each outcome may differ between countries. ${ }^{10-14}$ 20-21 In Britain, there has been a long term decline in smoking prevalence in men and women. ${ }^{22}$ The prevalence of obesity has increased but average blood pressure has fallen and there is some evidence that cholesterol concentrations may have fallen during the 1990 s. $^{23}$

\section{TRENDS IN CHD PREVALENCE}

One important source for monitoring future trends in CHD prevalence in Britain will be the Health Survey for England, a cross sectional population survey carried out annually since 1991. A four year comparison (1991 to 1994) found no compelling evidence of a trend in angina symptoms (using the WHO (Rose) definition) or in self reported diagnosed CHD over this very short period. ${ }^{24}$ Recent results from the 1998 survey (the next to assess cardiovascular disease) showed no significant changes since 1994 in either measure. ${ }^{25}$ We know of no other population based analyses of time trends in CHD prevalence in Britain. There are some reports from other countries but these do not provide a clear or consistent picture of the effect of declining event rates on CHD prevalence. A US study analysed trends in the National Health Interview Survey and found that the prevalence of all coronary disease (based on self report of a $\mathrm{CHD}$ condition during the previous 12 months only) remained relatively stable overall from 1980 to 1989, although there was an increase among white men. ${ }^{26}$ However, the Reykjavik study found that in Icelandic middle aged men the prevalence of all CHD (symptomatic or silent MI, or Rose angina symptoms) fell from 1968 to 1986; this fall was driven by a marked decline in the prevalence of angina without MI. ${ }^{27}$ A Swedish MONICA substudy reported a significant decline in Rose angina symptom prevalence from 1986 to 1994 for women but not for $\mathrm{men}^{28}$; however, power was limited by small numbers. Our study highlights the importance of differentiating between current and cumulative (lifelong) measures of prevalence and suggests that in British middle aged men the prevalence of history of diagnosed CHD has not fallen in parallel with angina symptom prevalence.

\section{REASONS FOR PREVALENCE TRENDS}

While both measures of CHD prevalence used in this study depend on the interplay of incidence and survival, the prevalence of angina symptoms might be additionally affected by changes in the severity of symptoms and the presence of effective treatment (medical or invasive). When we repeated the angina symptom 
trend analysis, adjusting in addition for history of diagnosed $\mathrm{CHD}$ at each questionnaire, the annual decline in symptom prevalence became even more pronounced $(-2.6 \%)$, but it was found to be of a similar magnitude in those with and those without diagnosed CHD $(-2.7 \%$ v $-2.5 \%$, respectively; $\mathrm{p}=0.9$ for interaction). The fact that symptom prevalence has declined in men both with and without diagnosed CHD suggests that increased rates of effective treatment may well be playing a part, but that a true decline in the occurrence of new angina, and perhaps a decline in disease severity, are also likely to be important.

A lifelong measure of prevalence such as diagnosed CHD might be expected to change more slowly in response to trends in acute event rates as, once a diagnosis is acquired, it remains until death, regardless of treatment, changes in symptoms, and occurrence or absence of new events. It is also possible that several opposing changes have contributed to the stable prevalence of diagnosed CHD. In particular, while the rate of new major events has declined, the ascertainment of milder forms of CHD may have increased. There is evidence from our data to suggest that the threshold for diagnosis of angina fell over the time of the study: middle aged men with angina symptoms were more likely to report a doctor's diagnosis of angina in the 1990s than the 1980s (data not shown.) This suggests that angina is likely to be diagnosed more frequently and with greater confidence in Britain nowadays, perhaps because of greater availability of confirmatory diagnostic procedures and wider awareness of the potential for secondary prevention. This is supported by results from the general practice morbidity studies, which showed that the percentage of people with a recent consultation for angina increased greatly in 1991-92 compared with previous decades. ${ }^{29}$ Therefore, increased recognition and ascertainment of angina may partly explain why the prevalence of diagnosed CHD remained stable over the study period while the rate of new major events and the prevalence of angina symptoms fell.

SOCIAL CLASS AND CHD TRENDS

A recent UK National Heart Forum report claimed that the social class divide in coronary mortality and morbidity in Britain was widening. ${ }^{5}$ However, this result was driven largely by the absence of a mortality trend from 1970 to 1993 in social class V compared with clear falls in all other classes. We found that symptom prevalence and major event rates had declined in both manual and non-manual classes; they were not significantly larger in the non-manual group. Our study lacked power to detect small social class differences or to examine trends in social class V separately.

\section{CONCLUSIONS AND IMPLICATIONS}

The falling prevalence of angina symptoms in British middle aged men from 1978 to 1996 suggests a lessening burden of disability from $\mathrm{CHD}$, probably as a result of both improved treatment and a decline in the incidence of new angina. It is possible that this may eventually lead to a reduction in requirement for symptom relieving interventions such as coronary revascularisation, though the effects of this falling prevalence must be set against the current level of unmet need and a tendency for intervention thresholds to lower over time. ${ }^{29}$ However, the proportion of middle aged men with a history of diagnosed CHD has not changed discernibly; this prevalence measure will probably be slow to respond to declines in acute event rates, especially if increasing medical recognition of less serious CHD is one of the driving factors. The need for antiplatelet drugs, statins, and other secondary preventive treatments for middle aged men with a history of established CHD is as great as ever; meeting this need will require intensified and sustained efforts. Primary prevention strategies to bring about continued declines in CHD incidence also remain a major challenge. The British Regional Heart Study is a British Heart Foundation
Research Group and also receives support from the Department of Health. Fiona Lampe was funded by the Department of Health.

1 Charlton J, Murphy M, Khaw K, et al. Cardiovascular disease. In: The heath of adult Britain 1841-1994 vol 1; Decennial Suppl 13. London: The Stationary Office, 1997.

2 Tunstall-Pedoe H, Kuulasmaa K, Mahonen M, et al for the WHO MONICA (monitoring trends and determinants in cardiovascular disease) project. Contribution of trends in cardiovascular disease) project. Contribution of trends in mortality: 10-year results from 37 WHO MONICA project populations. Lancet 1999;353:1547-57.

3 Hunink MGM, Goldman L, Tosteson ANA, et al. The recent decline in mortality from coronary heart disease, recent decline in mortality from corong

4 Sans S, Kesteloot H, Kromhout D on behalf of the Task Force of the European Society of Cardiology on Cardiovascular Mortality and Morbidity Statistics in Europe. The burden of cardiovascular disease mortality in Europe. Eur Heart f 1997; 18:1231-48.

5 McPherson K, Dunnell K, Leon D, et al. Coronary heart disease and risk factors: current patterns and future trends. In: Looking to the future: making coronary heart disease an epidemic of the past. National Heart Forum. London: The Stationary Office, 1999

6 Shaper AG, Pocock SJ, Walker M, et al. British regional heart study: cardiovascular risk factors in middle-aged men in 24 towns. BMF 1981;283:179-86.

7 Walker M, Shaper AG, Lennon L, et al. Twenty year follow-up of a cohort based in general practices in 24 British towns. f Public Health Med 2000;22:479-85.

8 Cook DG, Shaper AG, Macfarlane PW. Using the WHO (Rose) angina questionnaire in cardiovascular epidemiol(Rose) angina questionnaire in card

9 Shaper AG, Cook DG, Walker M, et al. Recall of diagnosis Shaper AG, Cook DG, Walker M, et al. Recall of diagnosis
by men with ischaemic heart disease. Br Heart $f$ 1984;51:606-11.

10 Sigfusson N, Sigvaldason H, Steingrimsdottir L, et al. Decline in ischaemic heart disease in Iceland and change in risk factor levels. BMF 1991;302:1371-5.

11 Salomaa V, Miettinen H, Kuulasmaa K, et al. Decline of coronary heart disease mortality in Finland during 1983 to 1992: roles of incidence, recurrence, and case-fatality: The FINMONICA MI register study. Circulation 1996;94: 3130-7.

12 Osler M, Sorensen TIA, Sorensen S, et al. Trends in mortlaity, incidence and case fatality of ischaemic heart disease in Denmark, 1982-1992. Int f Epidemiol 1996;25:1154-61.

13 Sytkowski PA, D'Agostino RB, Belanger A, et al. Sex and time trends in cardiovascular disease incidence and mortality: the Framingham heart study, 1950-1989. Am f Epidemiol 1996;143:338-50.

14 Rosamond WD, Chambless LE, Folsom AR, et al. Trends in the incidence of myocardial infarction and in mortality due to coronary heart disease, 1987 to 1994 . N Engl f Med 1998;339:861-7.

15 Volmink JA, Newton JN, Hicks NR, et al. Coronary event and case fatality rates in an English population: results of the Oxford myocardial infarction incidence study. Heart 1998;80:40-4.

16 Zeiner-Henriksen T. Comparison of personal interview and postal inquiry methods for assessing prevalence of angina and possible infarction. F Chronic Dis 1972;25:433-40.

17 Rose G, McCartney P, Reid DD. Self-administration of a questionnaire on chest pain and intermittent claudication. Br f Prev Soc Med 1977;31:42-8.

18 Lampe FC, Walker M, Lennon LT, et al. Validity of a self-reported history of doctor-diagnosed angina. 7 Clin Epidemiol 1999;52:73-81. 
19 Walker M, Shaper AG, Cook DG. Non-participation and mortality in a prospective study of cardiovascular disease. $f$ Epidemiol Community Health 1987;41:295-9.

20 Kuulasmaa K, Tunstall-Pedoe H, Dobson A, et al, for the WHO MONICA project. Estimation of contribution of changes in classic risk factors to trends in coronary-event rates across the WHO MONICA project populations. Lancet 2000;355:675-87.

21 Tunstall-Pedoe H, Vanuzzo D, Hobbs $\mathrm{M}$, et al, for the WHO MONICA project. Estimation of contribution of changes in coronary care to improving survival, event rates, and CHD mortality across the WHO MONICA project populations. Lancet 2000;355:688-700.

22 Bennett N, Jarvis L, Rowlands $\mathrm{O}$, et al. Living in Britain: results from the 1994 General Household Survey. London: HMSO, 2000.

23 Boreham R, Erns B, Falaschetti E, et al. Risk factors for cardiovascular disease. In: Health Survey for England 1998 London: The Stationary Office, 1999.
24 Lampe F, Colhoun H, Dong W. Cardiovascular disease and respiratory conditions. In: Health Survey for England 1994 Vol 1. Findings. London: The Stationery Office, 1996.

25 Primatesta P. Prevalence of cardiovascular disease. In: Health survey for England 1998. London: The Stationary Office 1999.

26 DeStefano F, Merritt RK, Anda RF, et al. Trends in nonfatal CHD in the United States, 1980 through 1989. Arch Intern Med 1993;153:2489-94.

27 Sigurdsson E, Thorgeirsson G, Sigvaldason H, et al. Prevalence of coronary heart disease in Icelandic men 19681986. The Reykjavik study. Eur Heart f 1993;14:584-91.

28 Glader EL, Stegmayr B. Declining prevalence of angina pectoris in middle-aged men and women. A populationbased study within the northern Sweden MONICA project. F Intern Med 1999;246:285-91.

29 Royal College of General Practitioners, The Office of Population Censuses and Surveys, and The Department of Health. Morbidity statistics from general practice, Fourth National Study. London: HMSO, 1995.

\section{IMAGES IN CARDIOLOGY}

\section{Large coiled right ventricular outflow tract thrombus resolved after thrombolytic treatment}

An 84 year old man with chronic bronchiectasis was referred by a family doctor following a three day period of dyspnoea and exercise related central chest pain. On the day of admission he had dizzy spells and a short episode of syncope lasting a couple of minutes. On admission he had sinus tachycardia (115 beats/min). Heart sounds were dual with no additional murmurs and jugular venous pressure was elevated. On chest auscultation, expiratory wheeze was audible throughout lung fields but there were no crepitations. ECG showed right bundle branch block with left anterior fascicular block. Transthoracic echocardiogram performed in the emergency unit showed normal left ventricular size with preserved systolic function. The right ventricle was dilated with moderate to severely impaired systolic function. There was mild tricuspid regurgitation with the right ventricular systolic pressure estimated at $40 \mathrm{~mm} \mathrm{Hg}$.

There was a large coiled thrombus within the right ventricular (RV) cavity attached to the pulmonary valve (PV), (panels A and B below). The thrombus was highly mobile and was prolapsing through the pulmonary valve into the pulmonary artery. The thrombus dimensions were: width $1.0 \mathrm{~cm}$, length $7.6 \mathrm{~cm}$. The patient received immediate thrombolytic treatment using recombinant tissue plasminogen activator (r-tPA) 20 units intravenously at 30 minute intervals, and both symptoms and pulse oximetry improved initially. However, after two hours the patient again became symptomatic with pronounced hypoxia and intermittent systemic hypotension. At that time an intravenous infusion of streptokinase was recommended, 50000 units per hour over a 24 hour period. The patient made a slow but uneventful recovery. Transthoracic echocardiogram repeated the next day showed no signs of thrombus (panel C) and improved right ventricular systolic function. Warfarin treatment was initiated and the patient was discharged home after nine days. At one year follow up, the patient is symptom-free with no episodes of recurrent pulmonary embolism.

PRZEMYSLAW PALKA BONITA ANDERSON ALEKSANDRA LANGE ppalka@hotmail.com
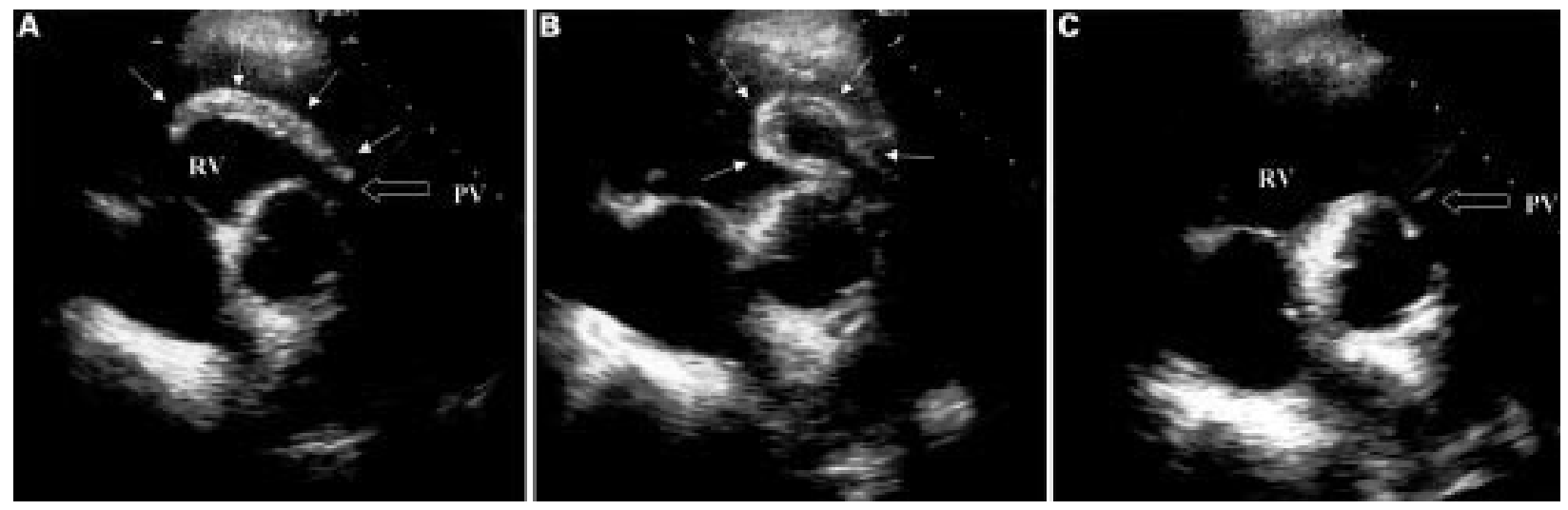